\title{
Fluxless Flip-Chip Bonding of Single Photodetector Chips Using Laser-Induced Forward Transfer
}

\author{
K.S. Kaur and G. Van Steenberge \\ Centre for Microsystems Technology, imec/Ghent University, Technologiepark 914A, B-9052 Gent, Belgium \\ email: Kaur.Kamalpreet@elis.ugent.be
}

\begin{abstract}
We demonstrate the successful flip-chip bonding and DC characterization of commercial photodiode chips via micro-bumps of indium and silver nano-particle (AgNP) based inks, printed using the Laser-Induced Forward Transfer (LIFT) technique.

OCIS codes: (130.6622) Subsystem integration and techniques; (140.3390) Laser materials processing; (200.4650) Optical interconnects
\end{abstract}

\section{Introduction}

There is an ever-increasing demand for miniaturization and in turn high-density circuits in the micro-electronics industry. In order to achieve this, efficient interconnection techniques are a pre-requisite. The existing interconnection techniques do not provide a combination of flexibility and high accuracy especially for fine-pitch bonding on a single chip scale. In this paper, we report the use of a laser-based bumping process called laser-induced forward transfer (LIFT) for flip-chip (FC) bonding of single photodiode (PD) array chips (schematic shown in fig. 1(a)). LIFT is a direct-write method for controlled printing of a wide range of materials and devices with micron and sub-micron resolution [1-3]. LIFT offers several advantages such as low temperature processing, flexibility, simplicity and high accuracy, thereby making it an excellent candidate as an interconnection technique.

\section{Experiments and results}

\subsection{Micro-bump transfer}

For the LIFT experiments, two different materials (donor) namely: indium and silver nano-particle (AgNP) based ink (U5603 from Sunchemicals) were used. Thin films of these materials were deposited onto glass substrates (carrier) employing evaporation and spin coating (3000 rpm, 20s) respectively. For the case of ink, after spincoating the donor samples were kept for $\sim 4$ hours under ambient conditions to achieve an optimum AgNP concentration and viscosity. The receiver comprised of glass substrates with $80 \mu \mathrm{m} \times 80 \mu \mathrm{m} \times 5 \mu \mathrm{m}$ lithographically patterned Ni-Au plated bond-pads. The donor-receiver assembly was then mounted on a X-Y translation stage and single laser pulses from a Timebandwidth Duetto $(355 \mathrm{~nm}, 12 \mathrm{ps})$ laser were then focused at the carrier-donor interface thereby, providing the thrust required to forward-transfer well defined micro-dots of the donor onto the receiver bond-pads. For the case of indium, six bumps were stacked on top of each other sequentially to achieve a bump thickness of $1.5 \mu \mathrm{m}$. All experiments were performed in air and at room temperature. Inset of fig. 1(b) shows the optical microscope image of a typical receiver substrate having micro-bumps laser-printed onto its contact pads.

\subsection{Flip-chip bonding of photodetectors}

Commercially available photodiode array $(1 \times 4)$ chips $\left(1 \times 0.450 \times 0.15 \mathrm{~mm}^{3}\right)$ from Albis Optoelectronics were then bonded to the LIFT assisted bumped receiver substrates using a semi-automatic flip-chip bonder (T-320X) from Tresky. Thermo-compression bonding technique was employed to establish an electrical and mechanical interconnection between the PDs and the substrates. The various key experimental parameters for LIFTing and bonding are depicted in the table below.

\subsection{DC characterization of the bonded assembly}

The FC bonded assemblies were then subsequently characterized both electrically and optically. A $980 \mathrm{~nm}$ LED source was used to illuminate the detector and a current/voltage source-meter unit (Keithley 2401) to reversely bias the PD and to read out the generated photocurrent. Figure 1 (b) shows the recorded photocurrent at different input powers as a function of applied bias voltage thereby, verifying the successful functioning of the PDs post-bonding. 
Table 1: Depicts the key LIFT and FC bonding parameters.

\begin{tabular}{|c|c|c|c|c|c|}
\hline $\begin{array}{c}\text { Donor } \\
\text { material }\end{array}$ & $\begin{array}{c}\text { Donor } \\
\text { thickness } \\
(\mu \mathrm{m})\end{array}$ & $\begin{array}{c}\text { Donor- } \\
\text { receiver } \\
\text { separation }\end{array}$ & Fluence & $\begin{array}{c}\text { Bump } \\
\text { dimensions } \\
(\text { dia, height })\end{array}$ & $\begin{array}{c}\text { Bonding pressure, } \\
\text { temp. } \\
\left.\text { (gf, }{ }^{0} \mathrm{C}\right)\end{array}$ \\
\hline Indium & 0.250 & in contact & $270 \mathrm{~mJ} / \mathrm{cm}^{2}$ & $20 \mu \mathrm{m}, 1.5 \mu \mathrm{m}$ & 100,157 \\
\hline AgNP ink & 5 & $50 \mu \mathrm{m}$ & $1.9 \mathrm{~J} / \mathrm{cm}^{2}$ & $20 \mu \mathrm{m}, 0.2 \mu \mathrm{m}$ & 100,150 \\
\hline
\end{tabular}

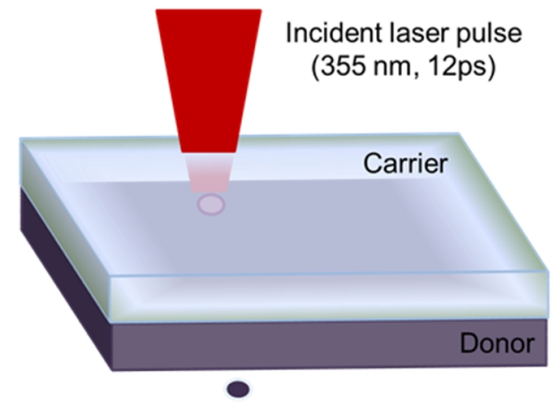

(a)

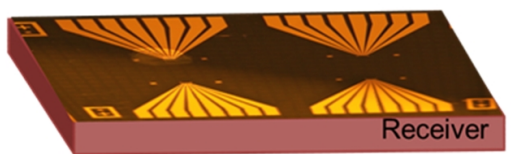



(b)

Fig. 1: (a): Illustration of the LIFT process, (b): I-V curves measured for the bonded assembly, inset: shows typical bumped receiver substrate and a PD chip bonded to it as viewed from the back-side.

\section{Conclusions}

In conclusion, LIFT-assisted thermo-compression FC bonding and DC characterization of single PD chips was successfully demonstrated using indium and AgNP based ink bumps. Experiments to determine the life-time of the bonded devices and their RF characterization are currently underway. The presented results show the great potential of the LIFT technique for high-density FC interconnections.

\section{Acknowledgement}

Part of this work was carried out in the framework of the "MIRAGE" project, funded within the European Commission FP7 program.

\section{References}

[1] J.Bohandy et.al, "Metal deposition from a supported metal film using an excimer laser", J.Appl. Phys. 6 (4), 1538-1539 (1986).

[2] K.S.Kaur et.al, "Waveguide mode filters fabricated using laser-induced forward transfer", Opt. Exp., 19 (10), $9814-9819$ (2011).

[3] K.S.Kaur et.al, "Flip-chip bonding of vertical-cavity surface-emitting lasers using laser-induced forward transfer", APL, 104 ( 6), 061102061102-3 (2014). 